\title{
Risk perception of NSAIDs in hospitalized patients in Greece
}

\author{
Karakitsiou $\mathrm{M}^{1}$, Varga $\mathrm{Z}^{2}$, Kriska $\mathrm{M}^{1}$, Kristova $\mathrm{V}^{1}$ \\ Institute of Pharmacology and Clinical Pharmacology, Faculty of Medicine, Comenius University, \\ Bratislava, Slovakia. mariakarakitsiou@hotmail.com
}

\begin{abstract}
BACKGROUND: Non-steroidal anti-inflammatory drugs (NSAIDs) are widely used analgesics worldwide in different syndromes. There is a relevant evidence about NSAIDs various adverse effects (AEs) on gastrointestinal, cardiovascular, renal, pulmonary, nervous systems. Many of these problems are preventable with respects to appropriate patient's risk perception.

OBJECTIVES: The main goal of our study was to examine drug risk perception with relation to participation factors as comorbidities in patients.

METHODS: A structured questionnaire was delivered to 124 patients hospitalized at Department of Internal Medicine in a selected General Hospital in Greece. Data were evaluated using a descriptive statistics.

RESULTS: Low awareness of NSAID risk was recorded, with $45.16 \%$ of respondents unaware of any particular AEs. Lack of this knowledge appears to be attributed to low communication of physicians and pharmacists with patients about possible risk from comorbidity, over half of respondents (55.8\%) had history of hypertension, and $25.9 \%$ were diabetics, which would increase the risk of NSAID therapy.

CONCLUSION: Our study revealed a restricted knowledge about risk of NSAIDs in the studied population and showed some important data related to the presence of comorbidity in patients, which could potentiate the risk of cardiovascular AEs (Fig. 5, Ref. 22). Text in PDF www.elis.sk.

KEY WORDS: non-steroidal anti-inflammatory drugs, risk perception, adverse effects, cardiovascular risk.
\end{abstract}

\section{Introduction}

Non-steroidal anti-inflammatory drugs (NSAIDs) belong to the most commonly used and prescribed drugs worldwide. They are extensively used to reduce the symptoms of pain and inflammation in several chronic diseases. In contrast, NSAIDs could potentially cause organ toxicity as gastrointestinal (1), cardiovascular $(2,3)$, renal $(4,5)$, respiratory (6), and nervous system toxicity (7). Recently, there have been reports of increased risk of thrombotic events such as: myocardial infarction and stroke during NSAID administration $(8,9)$. Moreover, NSAIDs and cyclooxygenase-2 (COX-2) inhibitors may increase systemic blood pressure in hypertensive patients and abolish effect of antihypertensive drugs $(10,11)$. Furthermore, there is an association between NSAID use and various tachyarrhythmias (12) and risk of bleeding (13). Many of the above mentioned AEs are preventable. For improvement of patient's safety, analysis of NSAID usage and assessment of their risk perception is needed to include evaluation of the known risk factors. In order to limit the influence of adverse effects on the

${ }^{1}$ Institute of Pharmacology and Clinical Pharmacology, Faculty of Medicine, Comenius University, Bratislava, Slovakia, and ${ }^{2}$ Florida Hospital Orlando, Internal Medicine Residency, Orlando, FL, USA

Address for correspondence: M. Karakitsiou, Institute of Pharmacology and Clinical Pharmacology, Faculty of Medicine, Comenius University, Spitalska 24, SK-813 72 Bratislava, Slovakia.

Phone: +00306979867255 health of patients, NSAID users should rather be well informed about the possible adverse effects for early detection and treatment discontinuation (14).

The aim of the present study was the evaluation of NSAIDs use in hospitalized patients in a rural General hospital in Greece by using a method of questionnaire.

\section{Methods}

We have preferentially used a structured questionnaire to assess NSAID use and their risk perception. The method of questionnaire is generally used in pharmacoepidemiology and pharmacovigilance because of its simplicity and low cost. Our questionnaire consisted of 13 questions covering subgroups of our subject: 4 semi-openended, 2 visual analogue scales and 7 closed. The questionnaire assessed frequency of NSAIDs usage, information about NSAIDs organ toxicity and finally examined the intensity of pain in relation to drug risk perception. Moreover, we investigated whether the patients were informed about the NSAIDs risk use by their physician or pharmacist.

Our study group included 124 patients using NSAIDs, who were hospitalized in the Department of Internal Medicine in the General Hospital in Greece during the summer 2015. The collected data were evaluated with methods of descriptive statistics.

The most important limitation for this study was the restricted number of patients and the use of a questionnaire for data gathering with the inherent limitations of questionnaire studies, including the 


\section{$427-430$}

possibility of the patients to give wrong answers. It is also necessary to recognize the possible impact of the hospital environment and the presence of other patients.

This study was authorized by the Ethical Committee of the hospital and the data were collected according to relevant law of the Hellenic Republic.

\section{Results}

This study was comprised of 124 patients with a slight predominance of women (women 67 vs. men 57). The average age was $57.12( \pm 15.49)$ years. A significant percentage of the respondents $(22.58 \%, \mathrm{n}=28)$ attended only primary school, although the percentage of those, who have academic level of education was also high $(24.19 \%, \mathrm{n}=30)$. Additionally, a high number of patients $(20.96 \%, \mathrm{n}=26)$ attended a secondary school, while $32.25 \%(n=40)$ attended a high school. According to our results, paracetamol was the predominantly used analgesic $(41.93 \%, \mathrm{n}=$ 52), while ibuprofen being the most widely used NSAID (29.03\%, $\mathrm{n}=36$ ) (Fig. 1).

A significant percentage $34.61 \%(n=43)$ used NSAIDs because of chronic diseases such as: inflammatory musculoskeletal

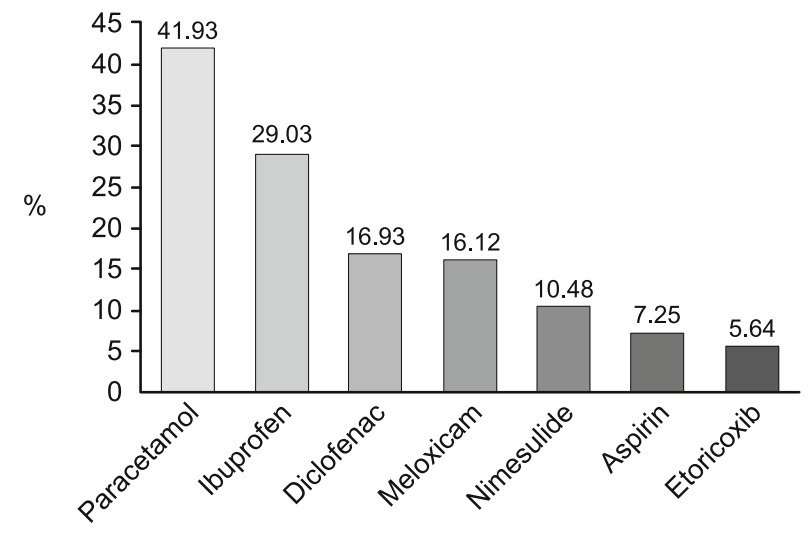

Fig. 1. Commonly used analgesics by respondents (percentage from the study group, $\mathrm{n}=124$ ).

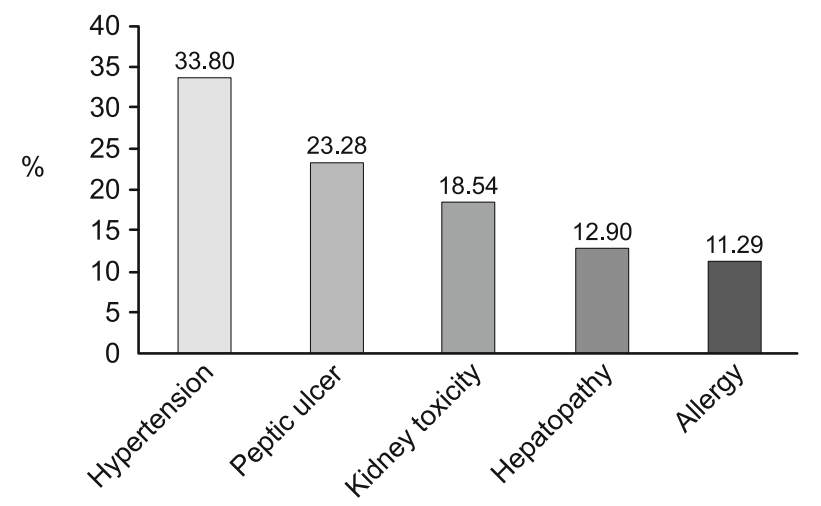

Fig. 2. Adverse effects recognized by the respondents (percentage from the study group, $n=124$ ).

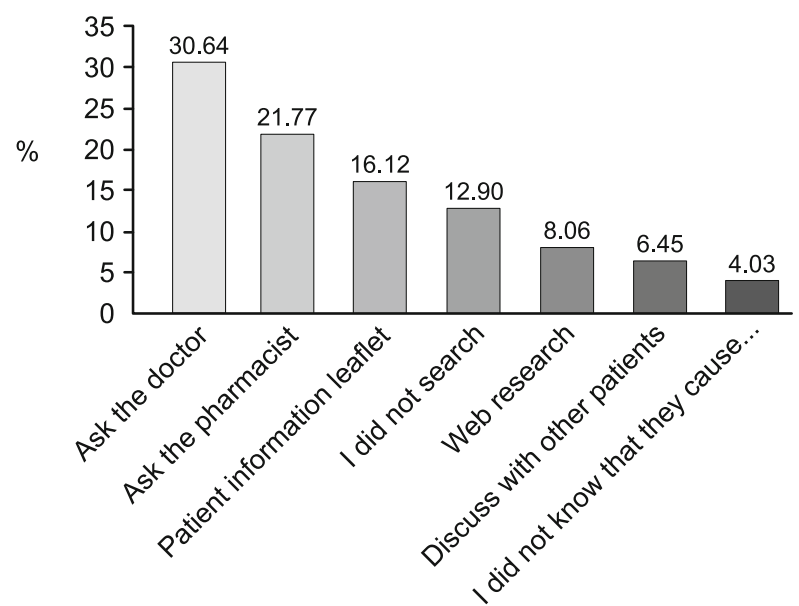

Fig. 3. Preferred sources of information about adverse effects (percentage from the study group, $n=124)$.

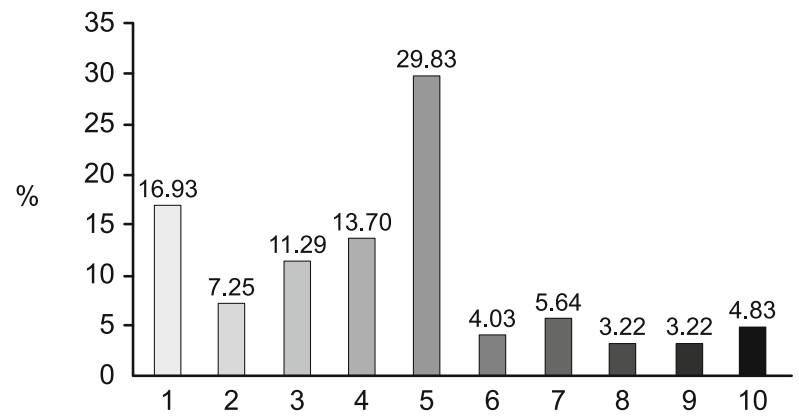

Fig. 4. Perception of risk of NSAIDs recorded in visual scale expressed as percentage of maximal value (10).

disorders (e.g. rheumatoid arthritis) and osteoarthritis. With regards to temporary sources of pain, $35.72 \%(n=44)$ used NSAIDs for headache, $6.8 \%(\mathrm{n}=8)$ for occasional joint pain and $3.45 \%(\mathrm{n}$ $=4)$ for backache. Only $2.13 \%(n=3)$ used NSAIDs for toothache. Some women referred that they used these drugs to relieve the pain during menstruation $(10.84 \%, \mathrm{n}=13)$.

Over half of the respondents $(54.83 \%, \mathrm{n}=61)$ mentioned that they were aware of at least some adverse effects of NSAIDs. On the other hand, $45.16 \%(n=56)$ referred that they did not know about any particular adverse effect, although they might have known that the treatment possesses some risks unknown to them.

When we asked the participants to define the adverse effects they knew about, most of them mentioned hypertension (33.8 \%, $\mathrm{n}=42$ ). In addition, the percentages of respondents were aware of the risk of peptic ulcer and kidney toxicity were also among the highest. At the same time, hepatopathy and allergic reaction were less recognized (Fig. 2).

The patients were asked whether they ever tried to gain information about potential adverse effects and about the means used to gather this information. Most of the respondents $(30.64 \%(n=38)$ asked their doctor, while $21.77 \%(n=27)$ asked their pharmacist. A significant percentage $(16.12 \%, n=20)$ read the Patient Infor- 


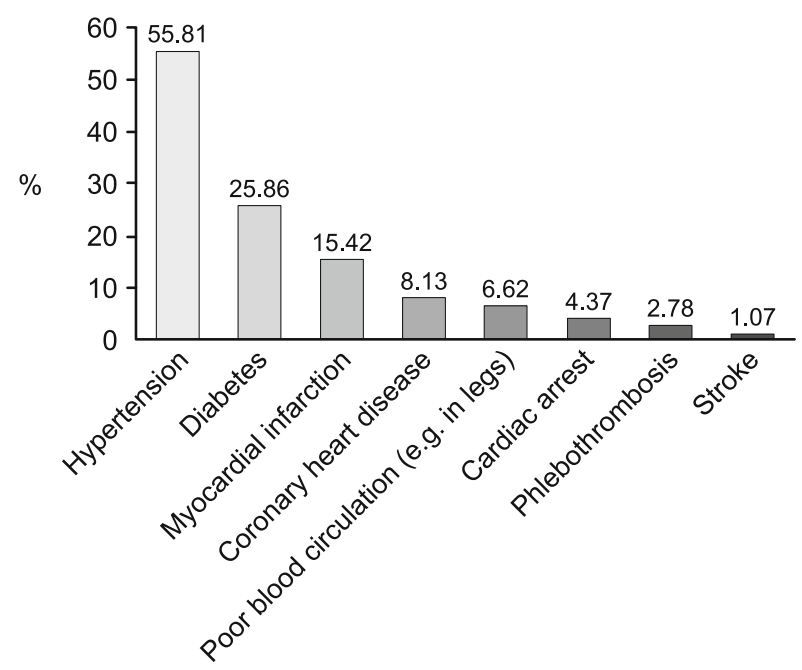

Fig. 5. Presence of comorbidities in a study group (percentage from the study group, $n=124$ ).

mation Leaflet. A web search was conducted by $8.06 \%(n=10)$, while $6.45 \%(n=8)$ of the respondents have discussed about the topic with other patients. Only $12.9 \%(n=16)$ referred that they have never done a survey about adverse effects of NSAIDs. However, $4.03 \%(n=5)$ declared that they did not know that NSAIDs can cause adverse reactions (Fig. 3 ).

Our respondents were also asked if their doctor informed them about the adverse reactions of NSAIDs spontaneously, without being solicited for this information. The majority of respondents $(63.48 \%, \mathrm{n}=79)$ were not informed by their doctor without a direct question. Nevertheless, $36.52 \%(n=45)$ of respondents have been informed spontaneously.

To participants were given a visual analogue scale to define their perception of risk posed by NSAIDs. The scale ranged from $1-10$ with 1 meaning that they are safe and 10 meaning they are very dangerous. The biggest group of patients $(29.83 \%, \mathrm{n}=36)$ defined the risk of NSAIDs as 5 according to our scale. It is important that $16.93 \%(\mathrm{n}=21)$ of patients consider these drugs totally safe, while only $4.83 \%(n=6)$ think that NSAIDs are exceedingly dangerous. The risk was estimated to be in the bottom half of the scale $(1-5)$ by $79 \%$ of respondents $(n=98)$ (Fig. 4$)$.

We also assessed the coexistence of certain diseases regarding the comorbidities of patients, which could constitute predisposing factors for the development of cardiovascular adverse effects of NSAIDs. Over half of our respondents suffered from hypertension $(55.81 \%, \mathrm{n}=69)$. There was also a significant percentage of diabetics $(25.86 \%, \mathrm{n}=32)$ or patients with history of a myocardial infarction (15.42\%, $\mathrm{n}=19$ ) (Fig. 5).

Simultaneous use of other, possibly interacting drug was also investigated. A great percentage of our respondents was receiving angiotensin converting enzyme (ACE) inhibitors $(63.2 \%, \mathrm{n}=78)$ and $\beta$-blockers $(59.8 \%, \mathrm{n}=74)$. A lower percentage was on antithrombotic treatment, the most widely used being clopidogrel (4.83\%, $\mathrm{n}=6)$, aspirin $(5.64 \%, \mathrm{n}=7)$ and warfarin $(5.64 \%, \mathrm{n}=7)$.

\section{Discussion}

The risk perception of NSAIDs was evaluated in this pilot study. Generally, our results from the Greek population are in an agreement with the data from different patient populations previously published in international papers. As reported by Adams, the frequency of use of NSAIDs was higher among patients with low educational level (15). The majority of our patients attended elementary or high school only, without subsequent university education.

Concerning the spectrum of NSAIDs, the most widely used analgesic among our patients was paracetamol and the second was ibuprofen. This is in agreement with the findings of Hudec et al (16) in the Slovak population. It is important to mention that diclofenac and etoricoxib were also often used by our study group. These drugs demonstrate high cardiovascular risk $(17,18)$. The study of McGettigan and Henry (19) found that diclofenac and etoricoxib together accounted for one third of all analgesics sold.

A significant percentage of our respondents used NSAIDs for chronic musculoskeletal disorders or headache. Pain relief during menstruation was also a common cause of NSAID administration. These results are in an agreement with the study of Wolfe et al (20).

With regards of possible adverse effects, the most widely recognized by our respondents were hypertension, peptic ulcer and kidney toxicity. These findings are comparable with the results of Cullen et al (21) and are similar to the results from a Slovak cohort obtained by Varga et al (14), although in our Greek study group, a much higher percentage of respondents knew about the risk of increased blood pressure. Only one out of four respondents knew that the risk of ulcers is particularly concerning. The most likely reason for the low information level of knowledge might be the fact that only every third respondent received information from the doctor or pharmacist about the risks of the therapy. As was reported earlier, also in the Slovak population a low number of physicians and pharmacists inform their patients spontaneously about the adverse effects of NSAIDs (14). This problem is most likely an international one, because doctors do not spend enough time with each patient for a high patient loads in their offices.

The majority of the patients ( $79 \%$ ) perceive the risk of NSAIDs as 5 or less on our visual analogue scale. In average, our study group estimated the risk of NSAIDs to be 4.36 $( \pm 2.41)$, which is in agreement with the results of Varga et al in the Slovak population (14).

The presence of certain patient's comorbidities in the clinical history of patients considerably increases the risk of cardiovascular adverse effects and is consequently considered to be the indicator of increased cardiovascular risk of NSAIDs. More than half of the study group had at least one of these diagnoses present, most commonly arterial hypertension. Every hypertensive patient was treated with antihypertensive drugs. Our findings agree with the results of Varga et al (14), who found that nearly $60 \%$ of NSAID users had arterial hypertension and documented antihypertensive treatment in $65.1 \%$ of NSAID users. The most widely used antihypertensive drugs in our study group were drugs with a high risk of decrease in antihypertensive effect when co-administered with NSAIDs (22). 
$427-430$

Patients with a high cardiovascular risk as: myocardial infarction, coronary heart disease and diabetes mellitus were also frequently present in our study. Our results suggest a relatively high risk of cardiovascular adverse effects in NSAID users hospitalized in the Department of Internal Medicine.

In conclusion, we recorded an alarmingly low awareness of the potential risks of treatment with NSAIDs. The lack of knowledge about possible adverse effects appears to be caused by the small number of physicians and pharmacists trying to directly inform the users of NSAIDs about the possibility of drug-related toxicity. Additionally, we found the presence of selected diagnoses in the histories of patients, which can raise the risk of cardiovascular adverse effects. This finding underscores the importance of proper patient education by healthcare professionals. In the context of adverse interactions, it seems necessary to consider the interaction potential of NSAIDs more thoroughly during the clinical decision making process. Our findings correlate with findings of authors from different countries, studying populations from different cultural background and healthcare delivery system. This illustrates the global, international nature of the problems studied.

\section{References}

1. Rybár J, Hlísta M, Masaryk P, Rovenský J, Hyrdel R, Kriska M. Gastroduodenal complications associated with non-steroidal antiinflammatory drugs in Slovak Republic: results of one-year prospective study. Vnitr Lek 2006; 52 (7-8): 673-676.

2. Gislason GH, Jacobsen S, Rasmussen JN et al. Risk of death or reinfarction associated with the use of selective cyclooxygenase- 2 inhibitors and nonselective non-steroidal antiinflammatory drugs after acute myocardial infarction. Circulation 2006; 113 (25): 2906-2913.

3. Gunter BR, Butler KA, Wallace RL, Smith SM, Harirforoosh S. Nonsteroidal anti-inflammatory drug-induced cardiovascular adverse events: a meta-analysis. J Clin Pharm Ther 2017; 42: 27-38.

4. Lipworth L, Abdel-Kader K, Morse J et al. High prevalence of nonsteroidal anti-inflammatory drug use among acute kidney injury survivors in the southern community cohort study. BMC Nephrol 2016; 17 (1): 189.

5. Lafrance JP, Miller DR. Selective and non-selective non-steroidal antiinflammatory drugs and the risk of acute kidney injury. Pharmacoepidemiol Drug Saf 2009; 18: 923-931.

6. Jenkins C, Costello J, Hodge L. Systematic review of prevalence of aspirin induced asthma and its implications for clinical practice. BMJ 2004; 328: 434.

7. Bigal ME, Serrano D, Buse D, Scher A, Stewart WF, Lipton RB. Acute migraine medications and evolution from episodic to chronic migraine: a longitudinal population-based study. Headache 2008; 48 (8): $1157-1168$.
8. Olsen AM, Fosbøl EL, Lindhardsen J et al. Long-Term Cardiovascular Risk of NSAID Use According to Time Passed After First-Time Myocardial Infarction: A Nationwide Cohort Study. Circulation 2012; 126 (16): 1955-1963.

9. Park K, Bavry AA. Risk of stroke associated with nonsteroidal antiinflammatory drugs. Vasc Health Risk Manag 2014; 10: 25-32.

10. Armstrong EP, Malone DC. The impact of nonsteroidal anti-inflammatory drugs on blood pressure, with an emphasis on newer agents. Clin. Ther 2003; 25: 1-18.

11. Solomon DH, Schneeweiss S, Levin R, Avorn J. Relationship between COX-2 specific inhibitors and hypertension. Hypertension 2004; 44: $140-145$.

12. Zhang J, Ding EL, Song Y. Adverse effects of cyclooxygenase-2 inhibitors on renal and arrhythmia events: Meta-analysis of randomized trials. JAMA 2006; 296: 1619-1632.

13. Ross SJ, Elgendy IY, Bavry AA. Cardiovascular safety and bleeding risk associated with nonsteroidal anti-inflammatory medications in patients with cardiovascular disease. Curr Cardiol Rep 2017; 19 (1): 8. doi: 10.1007/s11886-017-0814-5.

14. Varga Z, Kriska M, Kristova V, Petrova M. Analysis of non-steroidal anti-inflammatory drug use in hospitalized patients and perception of their risk. Interdiscip Toxicol 2013; 6 (3): 141-144.

15. Adams RJ, Appleton SL, Gill TK, Taylor AW, Wilson DH, Hill CL. Cause for concern in the use of non-steroidal anti-inflammatory medications in the community - a population-based study. BMC Family Practice 2011; $12: 70$.

16. Hudec R, Kriska M, Bozekova L, Foltan V. Comparison of NSAID consumption in Slovakia, Finland and Norway. Bratisl Lek Listy 2008; 109 (8): 370-373.

17. Trelle S, Reichenbach S, Wandel S et al. Cardiovascular safety of non-steroidal anti-inflammatory drugs: network meta-analysis. BMJ 2011; 342: c7086. doi: 10.1136/bmj.c7086.

18. Vonkeman HE, Brouwers JR, van de Laar MA. Understanding the NSAID related risk of vascular events. Brit Med J 2006; 332 (15): 895-898.

19. McGettigan P, Henry D. Use of non-steroidal anti-inflammatory drugs that elevate cardiovascular risk: an examination of sales and essential medicines lists in low-, middle-, and high-income countries. PLoS Med 2013; 10 (2): e1001388.

20. Wolfe F, Zhao S, Lane N. Preference For Nonsteroidal Anti-inflammatory Drugs Over Acetaminophen by rheumatic disease patients,A Survey of 1,799 Patients with Osteoarthritis, Rheumatoid Arthritis, and Fibromyalgia. Arthritis Rheum 2000; 43: 378-385.

21. Cullen G, Kelly E, Murray FE. Patients' knowledge of adverse reactions to current medications. Br J Clin Pharmacol 2006; 62 (2): 232-236.

22. Johnson AG, Nguyen TV, Day RO. Do nonsteroidal anti-inflammatory drugs affect blood pressure? A meta-analysis. Ann Intern Med 1994; 121: $289-300$. 